\title{
Synthesis and Evaluation of Stearylated Hyaluronic Acid for the Active Delivery of Liposomes to Liver Endothelial Cells
}

\author{
Naoyuki Toriyabe, Yasuhiro Hayashi, Mamoru Hyodo, and Hideyoshi Harashima* \\ Graduate School of Pharmaceutical Sciences, Hokkaido University; Sapporo, Hokkaido 060-0812, Japan. \\ Received February 18, 2011; accepted April 1, 2011; published online April 12, 2011
}

\begin{abstract}
Hyaluronic acid (HA) is a naturally-occurring ligand that can be useful for targeting liver endothelial cells. We describe herein the development of a new HA-lipid conjugate for the efficient delivery of liposomes to liver endothelial cells. When free HA coated cationic liposomes were injected into mice, their accumulation in the liver was significantly decreased depending on the content of free $\mathrm{HA}$, while accumulation in the lung was not significantly changed. When cationic liposomes modified with HA-stearylamine (HA-SA conjugate) were injected in mice, liver accumulation was increased depending on the amount of HA-SA conjugate and accumulation in the lung was drastically reduced, compared to non-modified liposomes. Confocal imaging analyses showed that HASA modified liposomes were accumulated to a greater extent along with blood vessels than non-modified liposomes, suggesitng that HA-SA modified liposomes are distributed in endothelial cells in the liver. Collectively, these findings indicate that an HA-SA conjugate is a useful material that can be used to modify liposomes and for delivering bioactive liposomal cargoes to liver endothelial cells.
\end{abstract}

Key words hyaluronic acid; liver endothelial cell; active targeting; liposome

It has recently been demonstrated that the dysfunction of liver sinusoidal endothelial cells is associated with liver diseases, such as liver fibrosis, cirrhosis, and portal hypertension, because the sinusoidal vascular network is disrupted. ${ }^{1)}$ It has also been reported that the defenestration of liver sinusoidal endothelial cells causes hyperlipidemia, because it is difficult for lipoproteins to reach hepatocytes, which was major cells involved in the uptake of lipoprotein. ${ }^{2)}$ Thus, a selective delivery system such as plasmid DNA (pDNA), short interfering RNA (siRNA), and drugs is important for the treatment of these sinusoidal endothelial cell-related diseases.

Hyaluronic acid (HA) is a naturally-occurring, biodegradable, biocompatible, non-immunogenic anionic biopolymer composed of alternating $\mathrm{N}$-acetyl-beta-D-glucosamine and beta-D-glucuronic acid residues linked by $1-3$ and $1-4$ linkages, respectively. HA is present a wide range of animal tissues from vertebrates to bacteria. ${ }^{3)}$ Although endogenous HA is continuously synthesized and degraded in the extracellular matrixs of almost all tissues, two major systems are responsible for its systemic removal and degradation. ${ }^{3,4)}$ Large HA molecules ( $\left.c a .10^{6} \mathrm{Da}\right)$ flow to lymph nodes, which remove and degrade $c a .85 \%$ of the HA, and the remaining small HA molecules ( $c a .10^{5} \mathrm{Da}$ ) enter the blood and are then cleared mainly by the liver endothelial cells. When exogenous hyaluronic acids are injected intravenously, they are rapidly removed from the blood and mainly accumulate in endothelial cells of the liver sinusoids, ${ }^{5,6)}$ because these cells express HA receptors such as cluster determinant 44 $(\mathrm{CD} 44),{ }^{7)}$ a receptor for hyaluronate-mediated motility (RHAMM),${ }^{8)}$ and the HA receptor for endocytosis (HARE). ${ }^{9}{ }^{9}$ For these reasons, hyaluronic acids are attractive biomaterials for drug delivery tools, because they are natural ligands and have desirable physicochemical properties.

HA has been widely used in the past as a targeted delivery material because of its carboxyl groups, which can form bonds with other molecules. Polycations such as poly-L-lysine $(\mathrm{PLL})^{10)}$ and polyethyleneimine (PEI) ${ }^{11,12)}$ were covalently bonded via amide bond formation between the amine groups of polycations and the carboxyl groups of HA, and these materials enabled the target-specific delivery of HA to receptor positive cells in vitro ${ }^{11,12)}$ and to liver endothelial cells in vivo. ${ }^{10)}$ Further, HA nanogels, in which thiol-conjugated HA was crosslinked via disulfide linkages, was used as a target specific siRNA delivery cargo. ${ }^{13)}$ While these materials are promising HA derivatives for delivering drug cargoes, optimization of the density of HA on the surface of the cargo is difficult because these cargoes could represent $100 \%$ of the HA on the surface. The characteristics of a cargo surface influences the interactions between ligands and receptors, as well as subsequent events, such as endosomal escape and release of the drug in the cytosol. ${ }^{14)}$ Thus, controlling the amount of ligand on the surface of the cargo is an important factor in the rational design of drug carriers. A liposome is a good cargo because the surface can be easily modified and controlled by introducing a ligand-lipid conjugate.

In the present study, an HA-stearylamine (HA-SA) conjugate, a new HA derivative, was synthesized the targeting ability of the HA-SA modified liposomes was evaluated. As a negative control, we used free HA coated liposomes to evaluate the effect of the lipid conjugate.

\section{MATERIALS AND METHODS}

Materials Dioleoylphosphatidylethanolamine (DOPE) and Cholesterol was purchased from Avanti Polar Lipids (Alabaster, AL, U.S.A.). $O, O^{\prime}$-Ditetradecanoyl- $N$-(alpha-trimethylammonio-acetyl)diethanolamine chloride (DC6-14) was purchased from Sogo Pharmaceutical (Tokyo, Japan). [Cholesteryl-1,2-3H(N)]-cholesteryl hexadecyl ether $\left.\left({ }^{3} \mathrm{H}\right] \mathrm{CHE}\right)$ was purchased from PerkinElmer Life Science Japan (Tokyo, Japan). Stearylamine, $\mathrm{N}, \mathrm{N}$-dimethylformamide (DMF), $\mathrm{N}$ hydroxysuccinimide (NHS), Chloroform, Deuterium oxide, DMSO- $d_{6}$ and tert-buthyl alchol were purchased from Wako Pure Chemicals (Osaka, Japan). 1-Ethyl-3-(3-dimethylaminopropyl)-carbodiimide hydrochloride (EDC) was purchased from Tokyo Chemical Industry (Tokyo, Japan). Hyaluronic acid (average molecular weight (MW) 500-700 
kDa) was purchased from Food Chemifa (Tokyo, Japan). Fluorescein labeled HA (average MW 600-1120 kDa) was purchased from PG Research (Tokyo, Japan). All other reagents were commercially available reagent-grade products.

Animals Male ICR mice (4-5 weeks old) and C57BL/6 mice (6-7 weeks old) were obtained from Japan SLC (Shizuoka, Japan). The experimental protocols were reviewed and approved by the Hokkaido University Animal Care Committee in accordance with the guidelines for the care and use of laboratory animals.

Hepatic Distribution of Injected Fluorescein-Labeled HA C57BL/6 mice were treated with $150 \mu \mathrm{g}$ fluorescein labeled HA in a $150 \mu \mathrm{l}$ phosphate buffered salts (PBS) solution by intravenous injection. The mice were killed $1 \mathrm{~h}$ after the treatment, and liver tissue samples were excised and fixed in $3 \%$ paraformaldehyde in PBS for $2 \mathrm{~h}$ at $4{ }^{\circ} \mathrm{C}$. The fixed samples were exposed to $20 \%$ sucrose in PBS overnight at $4{ }^{\circ} \mathrm{C}$. The samples were then embedded in Tissue Tek OTC compound (Sakura Finetek Japan Co., Ltd., Tokyo, Japan), and snap-frozen in liquid nitrogen. Cross sections of the liver samples $(16 \mu \mathrm{m}$ thickness) were incubated with $1.5 \%$ bovine serum albumin (BSA) dissolved in PBS at room temperature for $1 \mathrm{~h}$. The rat anti-mouse CD31 antibody (Biolegend) was used as primary antibody. The liver sections were then incubated with the primary antibody at $4{ }^{\circ} \mathrm{C}$ overnight. After washing with PBS, the sections were incubated with Alexa 568-labeled goat anti-rat immunoglobulin G (IgG) (Invitrogen) at room temperature for $1 \mathrm{~h}$. The samples were then observed by confocal laser scanning microscopy (LSM510 ver 3.2, Carl Zeiss Industrial Metrology, Oberkochen, Germany).

Cellular Uptake of Fluorescein Labeled HA Mouse primary liver endothelial cells and hepatocytes were prepared by the 2-step collagenase perfusion method and the Percoll gradient method as previously described, with minor modifications. ${ }^{15)}$ To obtain liver endothelial cells, non-parenchymal cells were incubated in a plastic dish for $15 \mathrm{~min}$ and the attached cells (kupffer cells) removed. The cell suspensions were incubated in fibronectin coated $35 \mathrm{~mm}$ dishes (IWAKI, Osaka, Japan) for $1 \mathrm{~h}$. In the case of hepatocytes, gelatin coated dishes were used and the cells were incubated for $4 \mathrm{~h}$ before use. After washing three times to remove unbound cells, $100 \mu \mathrm{g}$ fluorescein labeled HA was incubated with $1 \mathrm{ml}$ of culture medium, followed by a $3 \mathrm{~h}$ incubation at $37^{\circ} \mathrm{C}, 5 \%$ $\mathrm{CO}_{2}$. After washing the cells with PBS, the medium was changed to observation buffer (135 mM NaCl, $5.4 \mathrm{~mm} \mathrm{KCl}$, $1 \mathrm{~mm} \mathrm{MgCl}_{2}, 1.8 \mathrm{~mm} \mathrm{CaCl}_{2}, 5 \mathrm{~mm}$ 2-[4-(2-hydroxyethyl)-1piperazinyl]ethanesulfonic acid (HEPES), $10 \mathrm{~mm}$ glucose, $\mathrm{pH}$ 7.3) and samples were observed by confocal laser scanning microscopy (LSM510 ver 3.2, Carl Zeiss Industrial Metrology, Oberkochen, Germany).

Synthesis of the HA-SA Conjugate A $100 \mathrm{ml}$, onenecked, round-bottomed flask equipped with a magnetic stirring bar was charged with $10 \mathrm{mg}$ of hyaluronic acid (average MW $500-700 \mathrm{kDa})(16.6 \mathrm{nmol})$ in $25 \mathrm{ml}$ of water. $4.79 \mathrm{mg}$ of EDC $(25.0 \mu \mathrm{mol})$ and $2.88 \mathrm{mg}$ of NHS $(25.0 \mu \mathrm{mol})$ was added to the reaction mixture. The solution was stirred for $10 \mathrm{~min}$ and $20 \mathrm{mlof} \mathrm{DMF}$ and $5 \mathrm{ml}$ of chloroform were added. Next, $100 \mathrm{~mm}$ of a solution of stearylamine $(250 \mu \mathrm{l})$ in chloroform was added to the reaction mixture, which was then vigorously stirred overnight at $37^{\circ} \mathrm{C}$. The reaction mixture was dialyzed using a $5000 \mathrm{MWCO}$ dialytic membrane
(Spectrum Lab., CA, U.S.A.) against 11 of 10 times diluted PBS and tert-butyl alcohol $(\mathrm{v} / \mathrm{v}=1: 1)$ and water three times each. The solvents were removed with a centrifugal concentrator and the white solid was collected. The ratio for the incorporation of SA was determined by ${ }^{1} \mathrm{H}-\mathrm{NMR}$ using $\mathrm{D}_{2} \mathrm{O}$ and DMSO- $d_{6}$ as a solvent $(\mathrm{v} / \mathrm{v}=1: 1)$.

Preparation of Liposomes Liposomes were prepared by the hydration method. A lipid film was prepared in a glass test tube by evaporating an ethanol solution of lipids (137.5 nmol total lipids), composed of DC6-14, DOPE and cholesterol (molar ratio of DC6-14/DOPE/Chol was $40: 30: 30$ ). The prepared film was hydrated with $250 \mu$ l HEPES buffer, and the tube was then sonicated for $1 \mathrm{~min}$ in a bath-type sonicator (AU-25, AIWA Co., Tokyo, Japan). The free HA or HA-SA conjugate solution was then added to coat or introduce the conjugate on the surface of the liposomes.

Pharmacokinetics and Biodistribution Experiment in Mice Liposomes were prepared in which a non-exchangeable lipid label $\left[{ }^{3} \mathrm{H}\right] \mathrm{CHE}$ was incorporated. The labeled liposomes were injected via the tail vein into ICR mice. The injected volume was fixed at $10 \mu \mathrm{l}$ per $1 \mathrm{~g}$ of mouse weight. At the indicated times, the mice were sacrificed and the liver and lungs collected. After weighing the tissue samples, approximately $0.2 \mathrm{~g}$ of tissue samples were solubilized in $2 \mathrm{ml}$ of Soluene-350 overnight at $55^{\circ} \mathrm{C}$. All samples were decolorized by treatment with $\mathrm{H}_{2} \mathrm{O}_{2}$. Radioactivity was determined by liquid scintillation counting (LSC-6100; ALOKA Co., Ltd., Tokyo, Japan) after adding $10 \mathrm{ml}$ of scintillation fluid (Hionic fluor; Perkin Elmer, Inc., MA, U.S.A.) to the scintillation vials. Tissue accumulation was represented as the percent of injected dose (\%ID/tissue).

Visualization of the Hepatic Distribution of HA-SA Modified Liposomes Rhodamine labeled HA-SA modified liposomes composed of DC6-14, DOPE and cholesterol (molar ratio of DC6-14/DOPE/Chol/Rho-DOPE was $40: 30$ : $30: 1,416.6 \mathrm{nmol}$ total lipids) were used to visualize the liposome cargoes. The liposomes were modified with the HA-SA conjugate at a level of $1 \%$ nmol of total lipids. ICR mice were treated with Rhodamine labeled HA-SA modified liposomes by intravenous injection. The mice were killed 10 min after the treatment. The portal vein was cut and a needle was introduced into the vena cana and $10-15 \mathrm{ml}$ of heparin containing PBS (40 units $/ \mathrm{ml}$ ) solution was used to remove the remaining blood and cell surface bound liposomes in the liver. The liver was then excised and washed with saline and minced into small blocks $(10-15 \mathrm{~mm})$ with a scissors. The specimens were placed on a $35 \mathrm{~mm}$ glass base dish (IWAKI, Osaka, Japan) and observed by confocal laser scanning microscopy (Nikon A1; Nikon Co., Ltd., Tokyo, Japan).

Statistical Analysis Statistical comparisons were performed by one-way analysis of variance (ANOVA), followed by Student's $t$-test for two groups.

\section{RESULTS}

Confirmation of HA Localization in the Liver and Primary Cells To determine whether or not hyaluronic acid accumulates in liver endothelial cells, we investigated the extent of hepatic localization after an intravenous injection of HA. As shown in Fig. 1a, numerous signals from HA were detected in the liver sections. In addition, many of these sig- 
(a)

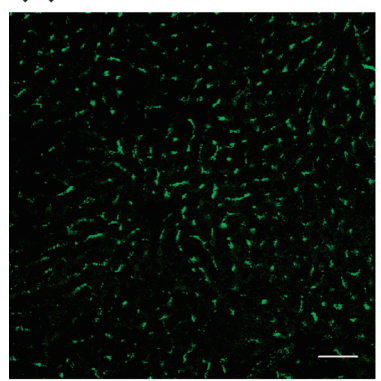

(d)

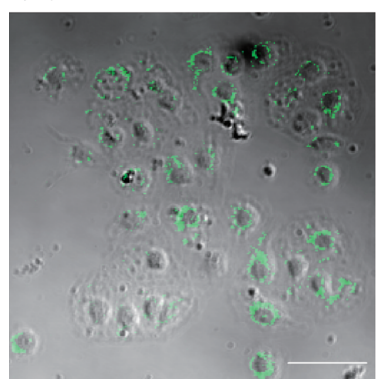

(b)

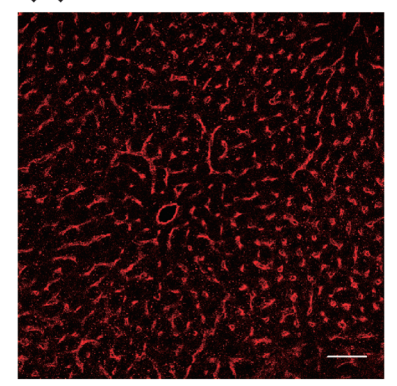

(e)

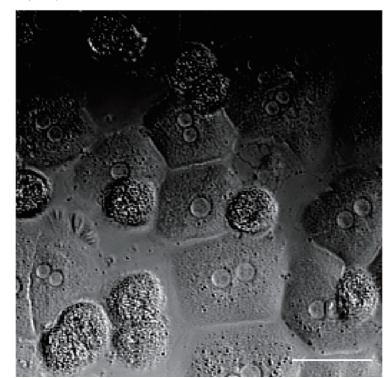

(c)

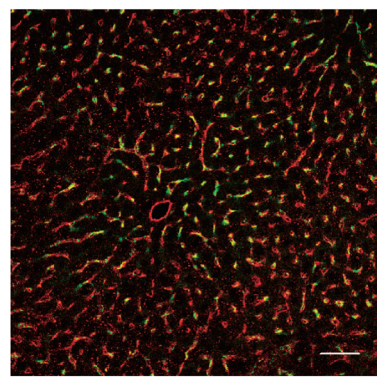

Mice were treated with fluorescein labeled HA via the tail vein for $1 \mathrm{~h}$ and liver tissue was analyzed. Green dots represent fluorescein labeled HA (a), red dots represent CD31 (b), and yellow dots represent the colocalization of HA and CD31 (c). Primary endothelial cells (d) and hepatocytes (e) were treated with fluorescein labeled HA for 3 h. Bar indicates $50 \mu \mathrm{m}$.

(a)

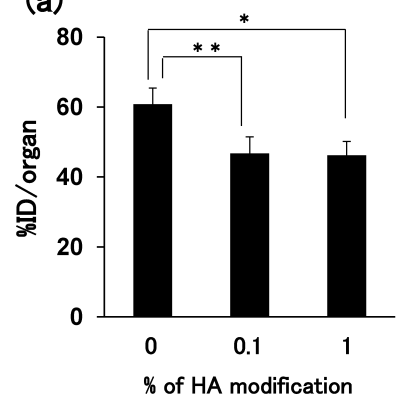

(b)

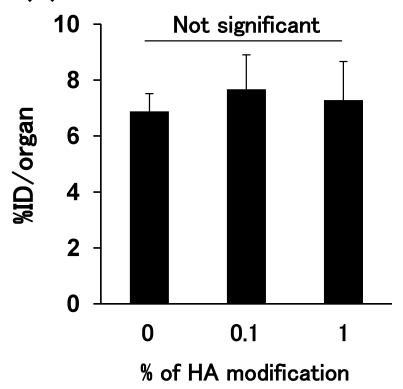

Fig. 2. Biodistribution of Free HA-Modified Liposomes in Mice

(a) Liver and (b) lung biodistribution of ${ }^{3} \mathrm{H}-\mathrm{CHE}$-labeled free HA coated liposomes in mice. Each mouse was treated by an injection of $10 \mu \mathrm{l}$ per g body weight and each tissue sample was collected $10 \mathrm{~min}$ after the treatment. Data are expressed as mean \pm S.D. $(n=3, * * p<0.01, * p<0.05)$.

nals were colocalized with CD31, which is a well known endothelial marker (Figs. 1b,c), indicating that a large fraction of the HA is distributed to liver endothelial cells. Next, the cellular uptake of HA was evaluated using mouse primary endothelial cells and hepatocytes. HA was internalized in the liver endothelial cells (Fig. 1d), while no signal was detected in the case of hepatocytes (Fig. 1e). Thus, HA indeed accumulates in and is internalized by liver endothelial cells.

Biodistribution of Free HA Coated Liposomes To determine whether or not free HA is actually displayed on the surface of liposomes and functions as a targeting ligand for liver endothelial cells, free HA was coated with cationic liposomes via electrostatic interactions. When the free HA coated liposomes were injected intravenously into mice, liver accumulation was significantly reduced (Fig. 2a), while lung accumulation was not significantly changed (Fig. 2b).

Synthesis and Characteristics of the HA-SA Conjugate

Hyaluronic acid is a polymer comprised of D-glucuronic acid and $N$-acetyl-D-glucosamine units connected by $\beta-1,3-$ or $\beta$ 1,4-glycosidic bonds. To prepare the HA-SA conjugate, we used the carboxylate moiety of D-glucuronic acid and conjugated it with the amino group of stearylamine (SA). SA was conjugated via an amide linkage using EDC and NHS as coupling agents and was incorporated randomly in the HA (Fig. 3a). After purification, we estimated the incorporation ratio of SA by ${ }^{1} \mathrm{H}-\mathrm{NMR}$ spectroscopy using the signal for the $\mathrm{N}$-acetyl group of HA $\left(\mathrm{NHCOCH}_{3}\right)$ and the methylene group of SA $\left(\mathrm{CH}_{3}\left(\mathrm{CH}_{2}\right)_{16} \mathrm{CH}_{2} \mathrm{NH}_{2}\right)$. We prepared several HA-SA conjugates by changing the incorporation ratio by controlling the amount of SA, EDC and NHS in the water-DMF- $\mathrm{CHCl}_{3}$ system. When we used a large excess (10 times equivalent to all carboxylic acid moieties of HA) of these reagents, it was possible to incorporate $100 \% \mathrm{SA}$ in the HA-SA conjugate, but it was poorly soluble in most solvents (water, a polar aprotic solvent like DMF and a nonpolar solvent like chloroform) and difficult to handle. After a number of tests, we finally used the HA-SA conjugate in which $18 \%$ SA was incorporated into the HA (Fig. 3b). This compound has a good solubility in water.

Pharmacokinetics and Biodistribution Studies of HASA Modified Liposomes The HA-SA conjugates were then used to introduce HA on the surface of liposomes. The physical properties of the HA-SA modified liposomes are shown in Table 1 . When HA-SA modified liposomes $(0.5 \%$ nmol of the total lipid) were injected intravenously, their accumulation in the liver was higher than the corresponding value for non-modified liposomes at every time point, as shown in Fig. 4a. It is noteworthy that the effect of HA-SA was significant $3 \mathrm{~min}$ after injection. On the other hand, lung accumulation was significantly reduced in the case of HASA modified liposomes (Fig. 4b). The influence of the 
(a)

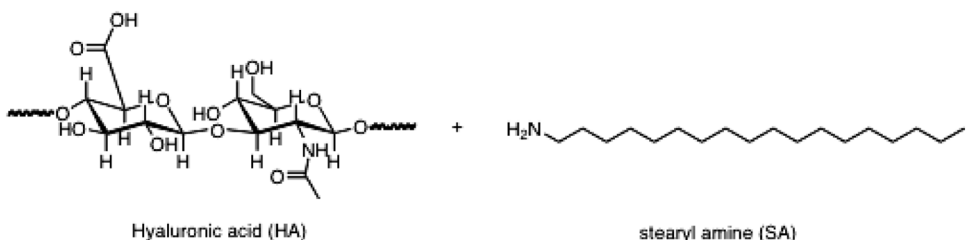

Hyaluronic acid (HA)

stearyl amine (SA)

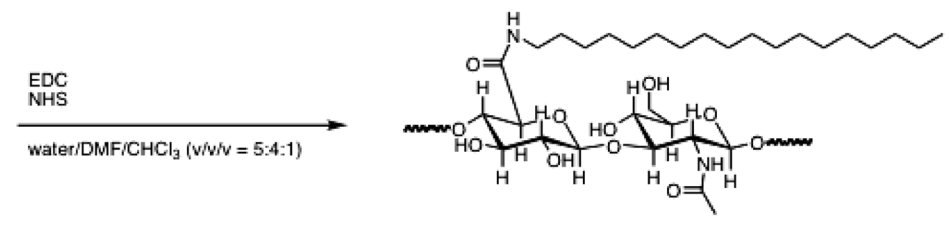

HA-SA conjugate
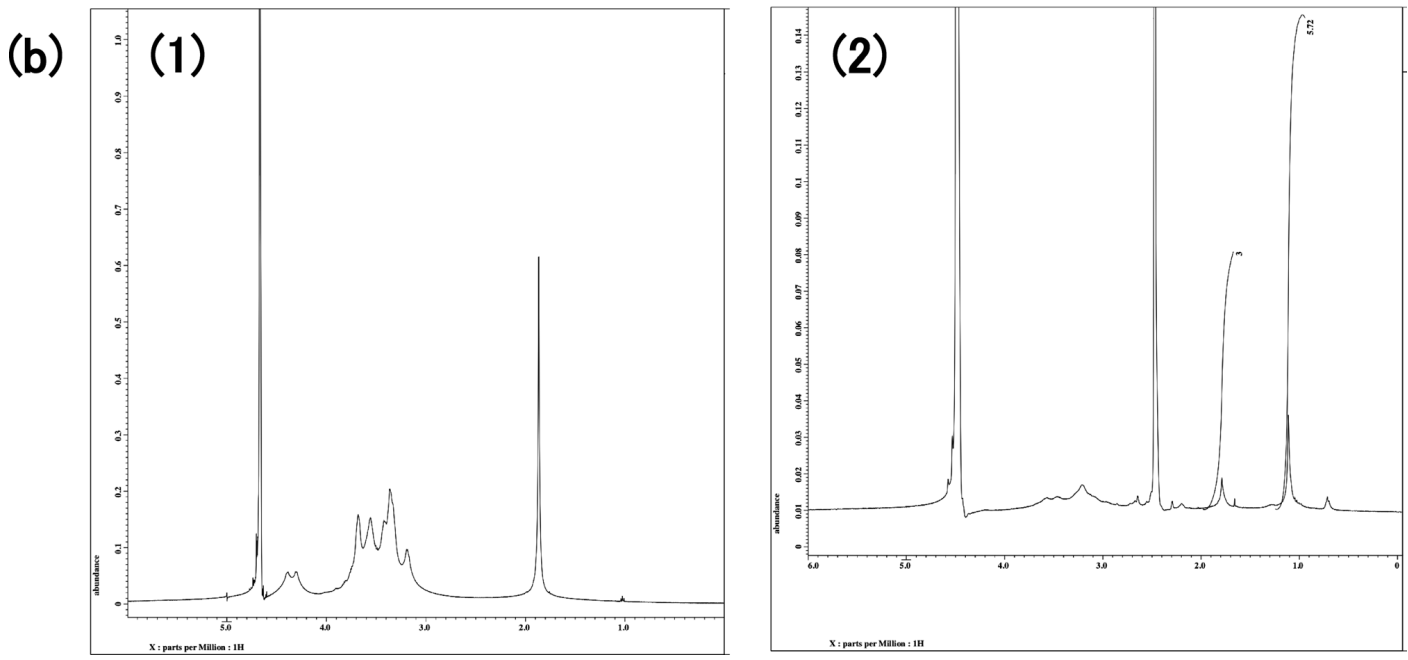

Fig. 3. Synthesis of the HA-SA Conjugate

(a) Schematic representation for the conjugation of hyaluronic acid (HA) with stearylamine (SA) using 1-ethly-3-(3-dimethylaminopropyl)carbodiimide (EDC). (b) ${ }^{1} \mathrm{H}$-nuclear magnetic resonance (NMR) spectra of HA measured in $\mathrm{D}_{2} \mathrm{O}(1)$ and HA-SA measured in $\mathrm{D}_{2} \mathrm{O} / \mathrm{DMSO}-d_{6}(\mathrm{v} / \mathrm{v}=1: 1)(2)$.

Table 1. Physical Properties of HA-SA Modified Liposomes

\begin{tabular}{llcc}
\hline \hline & $\begin{array}{c}\text { Size } \\
(\mathrm{nm})\end{array}$ & $\begin{array}{c}\text { Z-Potential } \\
(\mathrm{mV})\end{array}$ & PdI \\
\hline 0\% HA-SA liposome & $119 \pm 20$ & $57 \pm 10$ & $0.46 \pm 0.05$ \\
0.1\% HA-SA liposome & $159 \pm 15$ & $42 \pm 12$ & $0.35 \pm 0.01$ \\
0.5\% HA-SA liposome & $325 \pm 109$ & $8.1 \pm 27$ & $0.39 \pm 0.25$ \\
1\% HA-SA liposome & $254 \pm 19$ & $28 \pm 1$ & $0.26 \pm 0.04$ \\
2\% HA-SA liposome & $323 \pm 51$ & $27 \pm 3$ & $0.33 \pm 0.06$ \\
\hline
\end{tabular}

amount of HA-SA used in the modification was examined next. As shown in Fig. 4c, liver accumulation gradually increased depending on the extent of modification of HA-SA, whereas lung accumulation was significantly reduced in the case of a modification of 1\% (Fig. 4d).

Analysis of Hepatic Distribution of HA-SA Modified Liposomes Confocal microscopy analysis was performed to assess the targeting ability of the HA-SA modified liposomes to liver endothelial cells. The amount of modification of HA-SA was selected at $1 \%$ of total lipid, which showed a better pharmacological effect, as shown in Figs. 4c and d. HA-SA modified liposomes were accumulated to a greater extent along with blood vessels than non-modified liposomes (Figs. 5a, b). Next, the contribution of cellular uptake of liver endothelial cells was examined. The distribution pattern was similar in the case of liposomes that were unmodified or pretreated with an excess amount of free HA (Figs. 5a, c), however, the signals from HA-SA modified liposomes were reduced (Fig. 5d) in the case of a pre-treatment with an excess amount of free HA.

\section{DISCUSSION}

Our results provide the first demonstration that an HA-SA conjugate is a useful biocompatible material that enables the specific delivery of a cargo to liver endothelial cells in a short period of time, and that liposomes can easily be modified with it. In addition, the amount of conjugate on the liposome surface is easily controlled, which is necessary for further optimization to achieve additional selectivity and efficiency. This is discussed in more detail below.

Free HA accumulated in the liver endothelial cells after intravenous administration (Fig. 1a), and was internalized into the cells (Fig. 1d). These results are also supported by the fact that liver endothelial cells could take up HA both in vitro and in vivo. ${ }^{16)}$ Therefore, HA clearly has the ability to target liver endothelial cells. We next hypothesized that free HA coated liposomes could be accumulated to a greater extent in liver endothelial cells than non-coated liposomes, because free HA could be introduced on the surface of liposomes via 
(a)

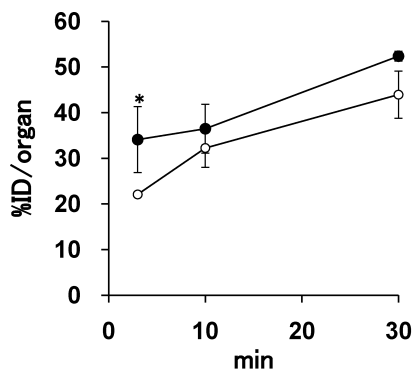

(c)

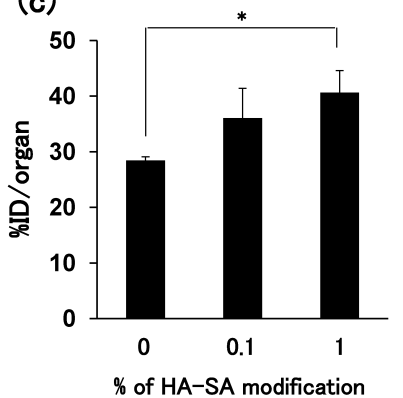

(b)

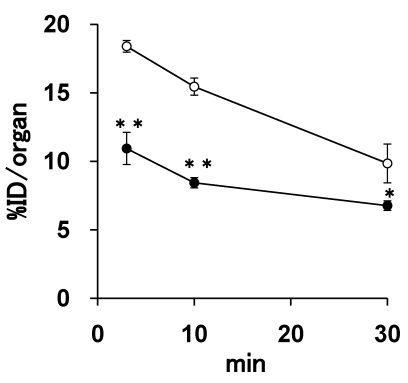

(d)

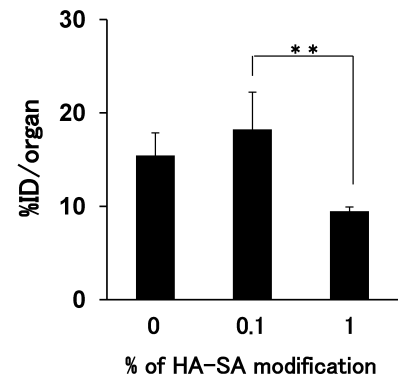

Fig. 4. Time Profiles and the Effect of HA-SA Modified Liposomes in Mice

Time profiles for (a) liver and (b) lung. Open circles and closed circles represen non-modified liposomes and HA-SA modified liposomes $(0.5 \% \mathrm{nmol}$ of total lipid), respectively. The effect of HA-SA modification in (c) liver and (d) lung. Each tissue was collected 10 min after intravenous injection. Data are expressed as mean \pm S.D. $(n=3$, $* * p<0.01, * p<0.05$ )

(a) non-modified liposome

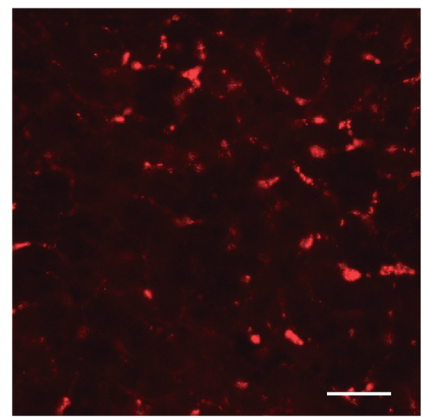

(c)non-modified liposome (free HA pre-treatment)

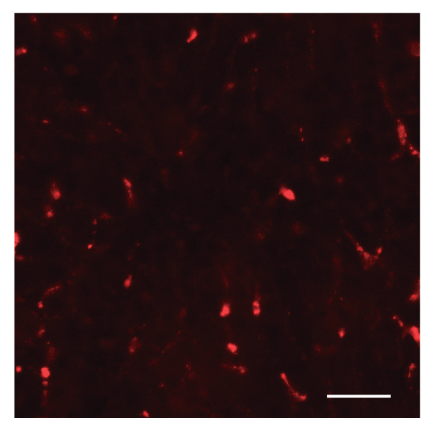

(b)HA-SA modified liposome

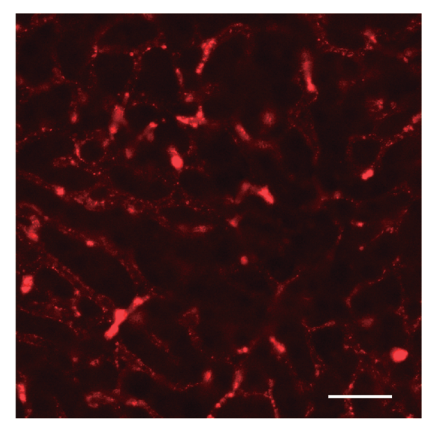

(d)HA-SA modified liposome (free HA pre-treatment)

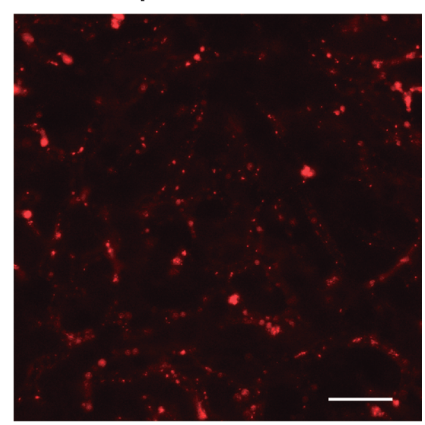

Fig. 5. Inhibiton of Liver Endothelial Cell Uptake by Pre-dosing with an Excess Amount of Free HA in Mice

Mice were treated with an excess amount of free HA ( $2.5 \mathrm{mg} /$ mouse $)$ for $3 \mathrm{~min}$. After the treatment, non-modified or HA-SA modified liposomes ( $1 \%$ of total lipid) labeled in Rhodamine-DOPE were injected in the mice, followed by incubation for $10 \mathrm{~min}$. The liver distribution pattern was analyzed by means of confocal microscopy. Bar indicates $50 \mu \mathrm{m}$. electrostatic interactions. Contrary to our expectations, the accumulation of free HA coated liposomes in the liver was decreased and this decrease was dependent on the amount of free HA (Fig. 2a). These results indicate that the reduction in positive charge on the surface of liposomes, as the result of modification with free HA leads to a decrease in accumulation in the liver. The reason why free HA did not fully function as a targeting ligand could be explained by fact that it is difficult to control the topology on the surface of a liposome, when free HA is used. Therefore, a better modification method (as an alternative to electrostatic interactions) that would allow HA to be appropriately displayed on the surface of liposomes is needed.

We focused on the HA-lipid conjugate because liposomes are easily modified with it via hydrophobic interactions between the lipid part of the conjugate and the liposomal surface so that HA could be appropriately displayed on the liposomal surface. Although a previous report demonstrated that many steps and a long time were required to prepare HA modified liposomes and the density of HA on the surface of the liposome was difficult to control, ${ }^{17)}$ this conjugate has an advantage, in that HA is introduced only by vortex and incubation with liposomes. We focused on stearylamine as a lipid linker because the stearylamine conjugate peptide (STR-R8) can be used to change the extent of modification on the surface of liposomes and this difference can effect the cellular uptake mechanism. ${ }^{18)}$ This suggests stearyalamine might be used to present HA on the surface of liposomes and would permit HA to function as a ligand.

When the HA-SA conjugate is mixed with liposomes, a $1 \mathrm{nmol} \%$ modification of total lipid is sufficient to coat the liposomal surface (Table 1). The zeta potentials of the free HA coated liposomes were similar (data not shown), but the pattern of their accumulation in the liver was different. HA-SA modified liposomes accumulated more heavily in the liver and less so in the lung over the short time (Figs. 4a, b). Contrary to the data shown in Fig. 2a, the accumulation of HASA modified liposomes was dependent on the amount of HA-SA present (Fig. 4c). These results suggest that, due to the lipid anchor, the HA is appropriately displayed on the surface of liposomes compared with free HA, and a large fraction of the HA-SA liposomes is taken up by the endothelial cells. The significant reduction in lung accumulation, as shown in Figs. $4 \mathrm{~b}$ and d, could be explained by the better topological effect of HA compared to the free HA, as shown in Fig. 2b. It is also possible that the negatively charged HA provides a shielding effect, because Ishiwata et al. demonstrated that the charge density on the surface of liposomes affects their accumulation by the lungs, ${ }^{19)}$ even though this contribution would be expected to be less. From confocal microscopy analyses, HA-SA modified liposomes accumulated along with blood vessels, and the accumulation was inhibited by pre-treatment with an excess amount of free HA (Figs. $5 \mathrm{~b}, \mathrm{~d}$ ), indicating that HA-SA modified liposomes could target HA receptors that are expressed on the surface of liver endothelial cells. However, some small, sharp signals along with the blood vessels remained, even when a pre-treatment was employed. These findings suggest that the origin of the signals is from uptake by kupffer cells. Mochizuki et al. reported that the cellular uptake of HA occurs in isolated kupffer cells, even though the contribution was about three times 
lower than that for endothelial cells. ${ }^{16)}$ Further studies are clearly needed for the more selective delivery to liver endothelial cells to be achieved by escaping uptake by kupffer cells. Optimization of the molecular weight of HA, and the use of a $\mathrm{pH}$ sensitive cationic lipid are some promising candidates. Other groups demonstrated that liposomes containing a HA-DOPE lipid conjugate had targeting ability for CD44 expressed cells in vitro, ${ }^{20,21)}$ however, these studies were only evaluated in vitro.

In conclusion, our study demonstrates that HA-SA can be useful in terms of ease of modification of a liposomal surface and efficient in vivo delivery to liver endothelial cells. To the best of our knowledge, this study is the first report of an evaluation of HA-lipid conjugate in vivo.

Acknowledgements This work was supported by the Special Education and Research Expenses of the Ministry of Education, Culture, Sports, Science and Technology of Japan. We also thank Dr. Milton Feather for editing this manuscript.

\section{REFERENCES}

1) Thabut D., Shah V., J. Hepatol., 53, 976-980 (2010).

2) Cheluvappa R., Denning G. M., Lau G. W., Grimm M. C., Hilmer S. N., Le Couteur D. G., Int. J. Infect. Dis., 14, e857-e867 (2010).

3) Fraser J. R., Laurent T. C., Laurent U. B., J. Intern. Med., 242, 27-33 (1997).

4) Weigel P. H., Yik J. H., Biochim. Biophys. Acta, 1572, 341-363 (2002).

5) Smedsrød B., Pertoft H., Eriksson S., Fraser J. R., Laurent T. C., Biochem. J., 223, 617-626 (1984).
6) Fraser J. R., Alcorn D., Laurent T. C., Robinson A. D., Ryan G. B., Cell Tissue Res., 242, 505-510 (1985).

7) Aruffo A., Stamenkovic I., Melnick M., Underhill C. B., Seed B., Cell, 61, 1303-1313 (1990).

8) Hardwick C., Hoare K., Owens R., Hohn H. P., Hook M., Moore D., Cripps V., Austen L., Nance D. M., Turley E. A., J. Cell Biol., 117, 1343-1350 (1992)

9) Zhou B., Weigel J. A., Fauss L., Weigel P. H., J. Biol. Chem., 275, 37733-37741 (2000).

10) Takei Y., Maruyama A., Ferdous A., Nishimura Y., Kawano S., Ikejima K., Okumura S., Asayama S., Nogawa M., Hashimoto M., Makino Y., Kinoshita M., Watanabe S., Akaike T., Lemasters J. J., Sato N., FASEB J., 18, 699-701 (2004).

11) Jiang G., Park K., Kim J., Kim K. S., Hahn S. K., Mol. Pharm., 6, 727-737 (2009).

12) Jiang G., Park K., Kim J., Kim K. S., Oh E. J., Kang H., Han S. E., Oh Y. K., Park T. G., Kwang Hahn S., Biopolymers, 89, 635-642 (2008).

13) Lee H., Mok H., Lee S., Oh Y. K., Park T. G., J. Controlled Release, 119, 245-252 (2007).

14) Petros R. A., DeSimone J. M., Nat. Rev. Drug Discov., 9, 615-627 (2010).

15) Yamauchi J., Hayashi Y., Kajimoto K., Akita H., Harashima H., Biol. Pharm. Bull., 33, 926-929 (2010).

16) Mochizuki S., Kano A., Shimada N., Maruyama A., J. Biomater. Sci. Polym. Ed., 20, 83-97 (2009).

17) Yerushalmi N., Margalit R., Arch. Biochem. Biophys., 349, 21-26 (1998).

18) Khalil I. A., Kogure K., Futaki S., Harashima H., J. Biol. Chem., 281, 3544-3551 (2006)

19) Ishiwata H., Suzuki N., Ando S., Kikuchi H., Kitagawa T., J. Controlled Release, 69, 139-148 (2000).

20) Surace C., Arpicco S., Dufaÿ-Wojcicki A., Marsaud V., Bouclier C., Clay D., Cattel L., Renoir J. M., Fattal E., Mol. Pharm., 6, 1062-1073 (2009).

21) Taetz S., Bochot A., Surace C., Arpicco S., Renoir J. M., Schaefer U. F., Marsaud V., Kerdine-Roemer S., Lehr C. M., Fattal E., Oligonucleotides, 19, 103-116 (2009). 